\title{
The Hennepin Ketamine Study Investigators' Reply
}

\author{
Jeffrey D. Ho; ${ }^{1,2}$ Jon B. Cole; ${ }^{1}$ Lauren R. Klein; ${ }^{1}$ Travis D. Olives; ${ }^{1}$ Brian E. Driver; ${ }^{1}$ Johanna C. Moore; ${ }^{1}$ \\ Paul C. Nystrom; ${ }^{1,2}$ Annie M. Arens; ${ }^{1}$ Nicholas S. Simpson; ${ }^{1,2}$ John L. Hick; ${ }^{1,2}$ Ross A. Chavez; \\ Wendy L. Lynch; James R. Miner ${ }^{1}$
}

\section{Hennepin Healthcare System, Department of Emergency Medicine, Minneapolis, Minnesota, USA \\ 2. Hennepin EMS, Minneapolis, Minnesota, USA

Correspondence:
Jeffrey D. Ho
Hennepin Healthcare System
Department of Emergency Medicine,
Minneapolis, Minnesota USA
E-mail: jeffrey.ho@hcmed.org

Conflicts of interest: none

Keywords: Hennepin; ketamine; research; sedation

\author{
Abbreviations: \\ AMSS: Altered Mental Status Scale \\ ED: emergency department \\ EMS: Emergency Medical Services \\ EFIC: Exception from Informed Consent \\ FDA: Food and Drug Administration \\ IND: Investigational New Drug \\ IRB: Institutional Review Board \\ LAR: legally authorized representative \\ WIC: Waiver of Informed Consent
}

Received: January 14, 2019

Accepted: January 19, 2019

doi:10.1017/S1049023X19000219
Ho JD, Cole JB, Klein LR, Olives TD, Driver BE, Moore JC, Nystrom PC, Arens AM, Simpson NS, Hick JL, Chavez RA, Lynch WL, Miner JR. The Hennepin Ketamine Study investigators' reply. Prehosp Disaster Med. 2019;34(2):111-113

We read with interest the recent editorial, “The Hennepin Ketamine Study,” by Dr. Samuel Stratton commenting on the research ethics, methodology, and the current public controversy surrounding this study. ${ }^{1}$ As researchers and investigators of this study, we strongly agree that prospective clinical research in the prehospital environment is necessary to advance the science of Emergency Medical Services (EMS) and emergency medicine. We also agree that accomplishing this is challenging as the prehospital environment often encounters patient populations who cannot provide meaningful informed consent due to their emergent conditions. To ensure that fellow emergency medicine researchers understand the facts of our work so they may plan future studies, and to address some of the questions and concerns in Dr. Stratton's editorial, the lay press, and in social media, ${ }^{2}$ we would like to call attention to some inaccuracies in Dr. Stratton's editorial, and to the lay media stories on which it appears to be based.

First, a brief overview of the study in question. This was a prospective, observational study of an EMS protocol change over a 12-month period regarding two medications commonly used for sedation within our EMS system: ketamine and midazolam. For the first six-month period, the EMS protocol recommended ketamine as a first line agent for sedation of agitated patients; for the second six-month period, midazolam was the recommended first line agent. There was no randomization. If a patient was deemed to need sedation based on the clinical judgment of the paramedic, the patient was given the medication per protocol. Importantly, no patients received medication for the sake of research; all sedatives were administered as part of usual clinical care. The only aspect of this work that was considered research was the careful observation of the effects of the sedative agents, and paramedic use of stopwatches to record the primary outcome (time to adequate sedation). We also recorded the frequency of adverse events.

Contrary to the Forbes report and Dr. Stratton's piece, our study was not approved under Exception from Informed Consent (EFIC; 21 CFR 50.24). Rather, the local Institutional Review Board (IRB) approved this study under Waiver of Informed Consent (WIC; 46 CFR 45.116). The two regulations are commonly confused. These regulations are similar in that they are both utilized in studies where informed consent is not possible, but are markedly different in their scope of practice and requirements. Our study was approved under WIC because it was deemed to be minimal risk by the IRB because it met all applicable criteria (shown in table). ${ }^{3}$ This study was deemed minimal risk because the only research interventions specifically related to the study were: (1) a stopwatch to measure time to adequate sedation; (2) the use of an agitation assessment scale (the Altered Mental Status Scale; AMSS); ${ }^{4}$ and (3) the use of a data collection form. Germane to the current study, research-related risks of these study types are typically limited to those of data collection and potential loss of confidentiality and do not usually include risks of the clinicallydetermined intervention. The US Food and Drug Administration (FDA; Silver Spring, Maryland USA) has provided guidance that "risks are not research-related when they are independent of the study and not associated with a trial intervention or protocol requirements." ${ }^{5}$ To additionally protect their rights, safety, and welfare, patients were notified of their enrollment in the study and given a mechanism to have data removed from the database.

The EMS personnel, when encountering agitated patients, were explicitly instructed to use their clinical judgment to assess the need for sedation. The agitation scale (AMSS) was used so paramedics could describe the conditions during which normal sedation practices 
took place in a standardized fashion; sedation was never mandatory for a given agitation score. While concern seems to exist that we may have lowered the threshold for sedation during the study periods, we explicitly instructed paramedics to use medication for sedation as they normally would. Internal EMS data support the notion that additional sedations did not take place during the study period. For example, in 2017, sedation for any behavioral disturbance occurred on $1.4 \%$ of EMS responses outside the study time period, and on $1.2 \%$ of EMS responses during the study time period.

Care for all patients in our EMS agency is dictated by our institutional EMS clinical treatment protocols, which by state statute are dictated by the EMS medical director. ${ }^{6}$ Both ketamine and midazolam have been standard treatments for agitation in our EMS system for many years (ketamine since 2008, midazolam since 2002), and analysis of our own peer-reviewed and published data suggests both drugs have similar risk profiles (including nearly identical intubation rates). ${ }^{7-9}$ As it was unclear if midazolam or ketamine was superior, changes in the first line recommendation, at the direction of the EMS medical director per state statute, were implemented so that each drug was the recommended first-line treatment for a six-month period, allowing for meaningful data collection to inform practice and improve care. During these periods, patients received the same medications whether data were collected for the study or not. This is not unlike any number of before/after EMS quality improvement projects, ${ }^{10}$ or recent groundbreaking work on intravenous fluid choices in the emergency department (ED). ${ }^{11}$ At all times, both midazolam and ketamine were available for use on our ambulances, and per usual EMS standard operating procedures, if the paramedics' clinical judgment was such that midazolam or ketamine was superior for a patient, they were allowed to administer whichever drug they deemed optimal for the patient. Our data reflect this is what actually occurred: during the study in the "ketamine first-line" time period, paramedics opted for another drug in 49 patients; during the "midazolam first-line" time period, paramedics opted for another drug in 62 patients.

We recognize the vulnerable nature of our patient population, and we have taken great care to ensure we have protected the rights, safety, and welfare of our patients. Over the years, we have sought to determine the optimal way to address consent in the patient population at-risk for agitation. Previously, we attempted to use a validated capacity to consent tool ${ }^{12}$ to determine if consent was even possible; however, when we studied this consent tool in 415 ED patients, only eight $(1.9 \%)$ had meaningful capacity to consent in the setting of alcohol intoxication. ${ }^{13} \mathrm{We}$ have also studied if a legally authorized representative (LAR) is commonly present to provide consent at the time prehospital sedatives are administered and found of 146 prehospital sedation encounters, in only nine cases $(6.2 \%)$ was a LAR present at the scene. ${ }^{8}$ Lastly, we screened nearly 1,500 patients at-risk for agitation in the ED to "pre-consent" for another agitation study, and were unable to enroll a single patient using this mechanism in a trial of two treatments for agitation. ${ }^{14}$

Based on available literature and expert opinion, it seems clear that meaningful informed consent in patients who are agitated or altered is not possible. ${ }^{13,15,16}$ Furthermore, in the context of severe agitation, obtaining consent before sedative administration would be unethical and dangerous, as research would have been placed ahead of clinical care. Some argue against research at all in this context in order to protect this vulnerable population. However, if all prospective research examining this patient population was halted, our collective approach as a specialty would rely on anecdote and retrospective chart reviews - both common sources of data that often produce inferior information. ${ }^{17,18}$ This would potentially result in poorer care delivered to these vulnerable patients. In fact, Dr. Stratton notes recent data suggest ketamine is gaining favor among EMS providers - this despite scant data to support its use. ${ }^{19,20}$

The EFIC studies are utilized to examine research questions related to "life-threatening conditions" where treatments are "experimental or unproven" when study subjects are unable to give informed consent, provided that five conditions are met, including community consultation for opinion and concern and filing the intervention as an Investigational New Drug (IND) with the FDA. ${ }^{3}$ We have previously used EFIC to study acute agitation in the $\mathrm{ED} ;{ }^{21}$ however, the FDA has recently determined patients with acute agitation in the ED (or prehospital) may not meet criteria for EFIC and subsequently declined requests for an IND for a study of patient-level randomization for several common agents in the ED and for a prehospital study.,22 In both of these instances, we conducted extensive community consultations with patients at high-risk for agitation (one of which was concurrent to the ketamine/midazolam study in question), which revealed substantial support for our work within our local community and the at-risk population. With local community and IRB support, we sought input from national experts on WIC and EFIC research and re-designed our studies as open-label observations of protocol changes.

The FDA has recognized that a gap exists in regulations regarding informed consent for patient populations that cannot provide consent, but are not experiencing a life-threatening condition. Although agitation can cause death when untreated, ${ }^{23}$ the FDA has recently declared that there is not sufficient evidence that agitation is a life-threatening condition. ${ }^{22}$ In 2016, the 21st Century Cures Act was passed, giving the ability to the FDA to grant an EFIC for randomized trials where the trials pose no more than minimal risk, though the exact mechanism for this has not been delineated. ${ }^{15}$ In July 2017, the FDA issued IRB guidance stating that until these regulations are finalized, FDA does not plan on objecting to local IRBs approving WIC studies provided they document the interventions are both standard of care and the study poses minimal risk. ${ }^{24}$ While this authority has only recently been explicitly granted, local IRBs have, occasionally, approved interventional randomized studies via WIC. In fact, Prehospital and Disaster Medicine published a randomized trial comparing haloperidol and midazolam for prehospital agitation in 2015 approved under WIC. ${ }^{25,26}$

Due to the undeserved negative media coverage, we elected to cease study data collection. The study was not suspended by the institution in a disciplinary manner; rather, the investigators voluntarily paused data collection in a good-faith effort to maintain the public trust for the benefit of our institution, community, and patients. The same medications continue to be available to EMS personnel for treatment of prehospital agitation; however, the cessation of data collection precludes a detailed accounting of the efficacy and complications of each drug. Unfortunately, these facts were not correctly represented by the media, and this appears to have influenced Dr. Stratton's editorial in the same way it influenced public opinion. Such incorrect representations serve to inflame public opinion but do little to protect vulnerable subjects or advance clinical care.

Maintaining the public trust is of paramount importance when it comes to WIC and EFIC research. We have learned much from 
our experience interacting with our community, the media, and colleagues in recent months. We are currently working to engage our community in all research more deeply than we have in the past. In the meantime, we hope that providing an accurate account of the study's methodology and consent provides clarity for our colleagues and our community in order to allow informed debate on the best approach to performing research in this important and largely under-studied aspect of emergency patient care.
References

1. Stratton SJ. The Hennepin Ketamine Study. Prehosp Disaster Med. 2018;33(5) 457-458.

2. Le Cong M. PHARM Podcast 208 Ketamine controversy with Minneapolis Police. PHARM: Prehospital and Retrieval Medicine. https://player.fm/series/prehospitaland-retrieval-medicine-podcast/pharm-podcast-208-ketamine-controversy-withminneapolis-police. Published July 31, 2018. Accessed December 5, 2018.

3. Klein L, Moore J, Biros M. A 20-year review: the use of Exception from Informed Consent and Waiver of Informed Consent in emergency research. Acad Emerg Med. 2018;25(10):1169-1177.

4. Miner JR, Klein LR, Cole JB, Driver BE, Moore JC, Ho JD. The characteristics and prevalence of agitation in an urban county emergency department. Ann Emerg Med. 2018;72(4):361-370.

5. US Department of Health and Human Services, Food and Drug Administration. Pregnant Women: Scientific and Ethical Considerations for Inclusion in Clinical Trials Guidance for Industry. https://www.fda.gov/downloads/Drugs/GuidanceComp lianceRegulatoryInformation/Guidances/UCM603873.pdf. Accessed December 22, 2018.

6. Minnesota Statutes 2018. Vol 144E.265 Subd 2. https://www.revisor.mn.gov/ statutes/cite/144E.265. Accessed December 22, 2018.

7. Martel M, Miner J, Fringer R, et al. Discontinuation of droperidol for the control of acutely agitated out-of-hospital patients. Prehosp Emerg Care. 2005;9(1):44-48.

8. Cole JB, Moore JC, Nystrom PC, et al. A prospective study of ketamine versus haloperidol for severe prehospital agitation. Clin Toxicol . 2016;54(7):556-562.

9. Miner JR, Heegaard W, Plummer D. End-tidal carbon dioxide monitoring during procedural sedation. Acad Emerg Med. 2002;9(4):275-280.

10. Malta Hansen C, Kragholm K, Dupre ME, et al. Association of bystander and first-responder efforts and outcomes according to sex: results from the North Carolina HeartRescue Statewide Quality Improvement Initiative. J Am Heart Assoc. 2018;7(18):e009873.

11. Self WH, Semler MW, Wanderer JP, et al. Balanced crystalloids versus saline in noncritically ill adults. N Engl J Med. 2018;378(9):819-828.

12. McCormack RP, Gallagher T, Goldfrank LR, Caplan AL. Including frequent emergency department users with severe alcohol use disorders in research: assessing capacity. Ann Emerg Med. 2015;65(2):172-177.e1.

13. Martel ML, Klein LR, Miner JR, et al. A brief assessment of capacity to consent instrument in acutely intoxicated emergency department patients. Am J Emerg Med. 2018;36(1):18-23.
14. Cole JB, Klein LR, Mullinax S, Nordstrom KD, Driver BE, Wilson MP. Study enrollment when "pre-consent" is utilized for a randomized trial of two treatments for acute agitation in the emergency department. Acad Emerg Med. 2018. E-pub ahead of print.

15. Dickert NW, Sugarman J. Ethics and regulatory barriers to research in emergency settings. Ann Emerg Med. 2018;72(4):386-388.

16. Eucker SA, Barrett TW, Schriger DL. Are two drugs better than one for acute agitation? A discussion on black box warnings, waiver of informed consent, and the ethics of enrolling impaired subjects in clinical trials. Ann Emerg Med. 2013;61(1):82-83.

17. Kaji AH, Schriger D, Green S. Looking through the retrospectoscope: reducing bias in emergency medicine chart review studies. Ann Emerg Med. 2014;64(3):292-298.

18. Lowenstein SR. Medical record reviews in emergency medicine: the blessing and the curse. Ann Emerg Med. 2005;45(4):452-455.

19. Mankowitz SL, Regenberg P, Kaldan J, Cole JB. Ketamine for rapid sedation of agitated patients in the prehospital and emergency department settings: a systematic review and proportional meta-analysis. J Emerg Med. 2018;55(5):670-681.

20. Buckland DM, Crowe RP, Cash RE, et al. Ketamine in the prehospital environment: a national survey of paramedics in the United States. Prehosp Disaster Med. 2018;33(1): 23-28.

21. Martel M, Sterzinger A, Miner J, Clinton J, Biros M. Management of acute undifferentiated agitation in the emergency department: a randomized double-blind trial of droperidol, ziprasidone, and midazolam. Acad Emerg Med. 2005;12(12): 1167-1172.

22. Klein LR, Driver BE, Miner JR, et al. Intramuscular Midazolam, Olanzapine, Ziprasidone, or Haloperidol for treating acute agitation in the emergency department. Ann Emerg Med. 2018;72(4):374-385.

23. Hick JL, Smith SW, Lynch MT. Metabolic acidosis in restraint-associated cardiac arrest: a case series. Acad Emerg Med. 1999;6(3):239-243.

24. IRB Waiver or Alteration of Informed Consent for Clinical Investigations. https:// www.fda.gov/downloads/RegulatoryInformation/Guidances/UCM566948.pdf. Accessed December 5, 2018.

25. Isenberg DL, Jacobs D. Prehospital Agitation and Sedation Trial (PhAST): a randomized control trial of intramuscular haloperidol versus intramuscular midazolam for the sedation of the agitated or violent patient in the prehospital environment. Prehosp Disaster Med. 2015;30(5):491-495.

26. Cole JB, Ho JD, Biros MH. Randomizing patients without consent: waiver vs exception from informed consent. Prehosp Disaster Med. 2016;31(4):457-458. 